\title{
Influence of Rules Change on Football
}

\author{
Jia You \\ School of Physical Education \\ Hubei University of Education \\ Wuhan, China 430205
}

\begin{abstract}
Football development cannot separate from change and improvement of rules. Football rules promote football development and influence football at the meantime guarantee the order on football field. Rules contribute to more scientific and reasonable football progress continuously. Contents, reasons and influence of change of rules in football match are analyzed through literature review method, case analysis method and information research method. Influence of evolution of match rules on football is analyzed from aspects such as fairness and safety of football match, skills and tactics.
\end{abstract}

\section{Keywords-football; match rules; influence; change}

\section{INTRODUCTION}

Modern football has had earth-shaking changes in continuous evolution since its appearance in 1863, and has become the most popular sport in the world. Learning football becomes prevailing in China, but people fail to understand rules and history of football, so inharmonious phenomenon appears.

Influence of evolution of match rules on football is analyzed through fairness and safety of football match as well as skills and tactics. Change of football rules closely relates to football development. Rules solve problems in matches and are timely and reasonably modified according to the development tendency of football, in order to promote sounder football development. Influence of important change of rules on football development is analyzed. The improvement of modern football rules will make football fairer, sounder, fiercer and more ornamental.

\section{RESEARCH OBJECT AND METHODS}

\section{A. Research Object}

Evolution history of football rules

\section{B. Research Methods}

1) Literature review method: Football rules having been researched by others on CNKI and literature are researched and analyzed.

2) Case analysis method: Events with significant change in football history are analyzed, such as the way of French team to take the crown on European Football Championship in 2000, responsibilities of football referee, the change of number of people and the change of Italian football.
3) Information research method: Football rules released by Federation International Football Association (FIFA) are understood through network, and are researched and analyzed.

\section{RESEARCH RESULTS AND ANALYSIS}

\section{A. Brief Description of FIFA Rules in the Football Competition}

1) Major events in the history of change of rules: The first football rule appeared in 1863; the concept of goal kick was first used in the competition of 1869; corner kick was added to football rules in 1872; FA installed beam on goal in 1875, and the rope always used before stepped down from the stage of history; a referee first whistled to hint foul in 1878, and afterwards, referees take whistle as tools; penalty kick was included in football rules in 1891; at the same time, net was installed on the goal; referees were allowed to referee in the competition field; FA set out-of-bounds area in competition field in 1902; different formations appeared in football match in 1958, and substitution of players was allowed but only the wounded can be substituted; red and yellow cards were first used in football match on Mexico World Cup in 1970; goalkeeper was forbidden to use hand to receive the ball back kicked by teammates in 1992 .

2) English football rules in 1863: Football field has length of 137.16 meters and width of 91.44 meters. The goal is constituted of two sticks without beam; the kick-off and goal are chosen through tossing a coin; if the ball is kicked out of the sideline, the opponent directly kicks the ball into the field and the match continues; players drive the ball home if the ball passes through the space between the two sticks, without the limitation of height; in the competition, body check is fair and legal, but players are forbidden to hug, push or kick and stumble others by feet; it is forbidden to make a racket in the competition.

3) Introduction to football rules: Self-improvement of match rules: Initially, there are only ten rules of modern football match and it is only used in England. With continuous development of football, old rules cannot meet demands of football development. It is improved after the establishment of FIFA. The organization responsible to modify and formulate football rules, namely International Football Association Board (IFAB) is specially set up. After modification and improvement, the number of football rules changes from ten to 
seventeen. Improvement of match rules guarantees fair and reasonable football match and promotes football development.

Application of favorable terms: In order to make the match more ornamental, reduce unnecessary waste of time and make the match more coherent, FIFA modified the provision in 1996. If the favorableness expected by the referee doesn't appear, the referee can penalize within the fixed time, no matter whether the referee gives favorable gesture.

\section{B. Positive Significance of Change of Rules on Football Match}

\section{1) Effects of modified football rules}

TABLE I. EFFECTS OF MODIFIED FoOTBALL RULES

\begin{tabular}{cl}
\hline Date & \multicolumn{1}{c}{ Modified rules } \\
\hline 1993 & $\begin{array}{l}\text { Give a warning through yellow card } \\
\text { to players who deliberately delay the } \\
\text { game, meanwhile the opponent } \\
\text { carries out indirect free kick in the } \\
\text { place of foul. }\end{array}$ \\
The referee will show the red card to \\
directly severely penalize those who \\
maliciously foul with vile nature.
\end{tabular}

1998 Standing on offside position doesn't foul. Players foul if they stand on offside position and obtain benefits.

2006 The referee may directly show the red card to penalize diving with vile nature.

Effects
$\begin{aligned} & \text { Make the match fluent and } \\ & \text { ornamental. }\end{aligned}$
Guarantee the safety of
players and encourage them
to break through and attack.

Emphasize defense and encourage attack, promote the development of attack tactics.

Guarantee fairness of competition at the meantime make the competition fiercer.

According to "Table I", the FIFA updates rules to solve problems that cannot be solved by previous rules in football match, so the improvement of rules is pertinent to promote football development.

2) Encourage attack: Modification of offside rule avails attackers and encourages players to strengthen attack, promoting continuous evolution of football tactics. In 1998, the modifications of offside rules include: it changes the rule that the referee will call the attacker offside if he stands on parallel line of the last but one defender; the attacker is not offside if he stands on the offside position but hints he will not participate in the attack; when attacker shoots, if the teammate stands on the offside position but doesn't obtain benefits, he cannot be called offside; the previous rules require the assistant referee to adhere to the rule of preferring misjudgment to neglected judgment on penalty of offside, while the new offside rules explicitly stipulate when the referee cannot judge, he should give the ball to the attacker. New offside rules aim at attack and make the competition fiercer.

Sudden death is used to promote players to strengthen attack and make the match more ornamental and more occasional. During the World Cup held in France in 1998, in overtime, players of both sides are completely exhausted. If the overtime of thirty minutes continues, players are unable to stand fatigue and it makes the match less splendid. The sudden death greatly stimulates players to strengthen attack to win, because it stipulates the match will be over immediately if the player of either side drives the ball home. It is difficult to predict victory or defeat during the penalty kick. It is one of the charms of football match.

3) Guarantee safety of players, completely eradicate violence and foul: In order to completely eradicate foul on football field, FIFA has formulated many provisions. During the France World Cup in 1998 and Japan and South Korea World Cup in 2002, FIFA sternly asks referees to show the red card if the player uses rude actions. It strengthens the protection of players and stimulates them to break through and attack in the match.

4) Reduce unnecessary waste of time and make the match more ornamental: According to the investigation of FIFA in 1993, the audiences are often strongly dissatisfied about the match of 90 minutes, because it wastes time and makes the match less ornamental. In order to change the situation, FIFA improves football rules that stipulate: If the goalkeeper fails to serve the ball after more than four steps, touches the ball for two times and makes his own team obtain benefits through using hands to receive the ball deliberately back kicked by teammates or deliberately delaying the game, the referee will penalize indirect free kick and even show the yellow card. The rule restricts behaviors of goalkeeper and promotes players to strengthen attack, so it makes the football match fiercer and more ornamental.

Players wounded in the match should be moved outside the field rapidly and be treated, and the match continues. The implementation of new rules reduces the waste of time. Each match has injury time to supplement the time consumed because of substitution of players or too far out-of-bound of balls.

The referee should seldom interfere in or interrupt the match. Before the implementation of new rules, if foul appears, the referee will penalize and frequently interrupt the match, making the match less fluent and ornamental. The situation is changed after the implementation of new rules. After foul appears, the referee can does not penalize or supplement the penalty after the attack. In other worlds, the referee doesn't give the penalty favorable for offending team. It prevents the waste of time and encourages attack.

Application of multiple ball system avoids the waste of time on picking up balls and makes the match more coherent. It is a part of rules modified by FIFA. Football rules aim at making the match just and fair. Its modification and improvement promote better development of football. However, its modification and improvement must be fair.

5) Guarantee the match is just and fair: The work system of referee is continuously reformed. In order to make the referee just and fair in judgment, and guarantee the match is smooth and splendid, the referee system has experienced numerous important reforms. The first match rule fails to explicitly stipulate responsibilities of referee. Referee can only appear outside the football field as. Responsibilities of referee were included in football rules in 1880 . Referees can enter the football field and use whistle to judge. After the offside rule 
appears, linesman appears and takes the flag to assist the referees. It is the work pattern of referees. Although the referee system has been used so far, its contents change greatly. There were only ten rules about referees before 1997, but no it has eighteen. Linesman has been transformed into assist referees since 1996 and their responsibilities increase significantly. Specific responsibilities are explicitly stipulated in the rules. The "sideline referee system" was gradually replaced by "diagonal referee system" in the 1950s. Nowadays, referees adhere to teamwork spirit. Many advanced science and technology are applied to tools of referees. Flags of assistant referees have wireless communication device. The implementation of rules reduces unnecessary errors of referees and makes the match fair and reasonable.

6) Promote the development of football skills and tactics: At first, people pay attention to attack in playing football, using the formation of "nine forwards and one back" and concentrating on attack. Although it promotes the development of attack tactics, it goes against football development in the long term. Therefore, when modifying rules, FIFA emphasizes offense-defense balance. Many new formations appear, such as "4-4-2", “4-3-3", "4-5-1”, "5-4-1" and " $3-4-3$ ", in order to make the formation more affluent.

Change of skills and tactics must be within allowance range of rules. Change of rules promotes new tactics. Three points system replaces two points system, having distinctly important effect on the development of football skills and tactics.

\section{CONCLUSION AND SUGGESTION}

\section{A. Conclusion}

New football rules promote football development. For example, the time that the goalkeeper holds the ball by hand is restricted to accelerate the match and make it more coherent and ornamental. The rule of encouraging goal promotes players to strengthen attack and makes the match fiercer and arouses the enthusiasm of audiences. FIFA World Cup is increasingly popular among people, indicating football enjoys increasing popularity. Change of rules is to enormously promote football development and ensure the match is fair and reasonable; guarantee health and safety of players and stop violence and foul on football field to exert skills of players; encourage attack to make football match ornamental; promote the development of football and its tactics. According to the development of football rules, rules guarantee and adapt to continuous development of football.

\section{B. Suggestion}

In continuous development, contemporary football encounters problems that cannot be solved by old rules, so the rules must be improved to adapt to football development. The formulation and improvement of rules must: insist justness and fairness, guarantee safety of players, promote the development of skills and tactics and make football match more ornamental. The modification of rules must proceed from the reality to continuously promote football development.
In formulation and modification of football rules, we must adhere to the principle of "take the essence and discard the dregs", inheriting scientific and reasonable old rules. Meanwhile, we must have creative spirit, namely innovate boldly under the premise of meeting demands of football development. In this way, the new rules formulated can constantly infuse new blood into football and make football always be "the world's first sport".

\section{REFERENCES}

[1] Football Contest Rule 2012 [M], Beijing People's Sports Press, 2012

[2] Zingerle. Research on Evolution Factors of Rules in Modern Football Match, Inner Mongolia Normal University, 2010

[3] Zhou Zhigang. Discussion on Origin, Feature Variation and Evolution of Rules of Football, Contemporary Sports Technology, 2011

[4] Zheng Mourong, Lyu Kangqiang. Analysis on Evolution of Rules in Football Match, Guangzhou Sport University, 2007

[5] Liu hongwei. Analysis on Influence of Change of Football Rules on Football Development, Theoretical Innovation, 2011 (8)

[6] Li Shupin. Looking New Development of Modern Football from Match Rules, Youth Literator, 2012

[7] Mu Wenming. Influence of Evolution of Match Rules on Football Development, Science and Technology Information (Adacemic Edition), October 2006 